\title{
Oligodendrocyte precursor cells transplantation protects blood-brain barrier in a mouse model of brain ischemia via $\mathrm{Wnt} / \beta$-catenin signaling
}

Liping Wang $\mathbb{1}^{1,2,3}$, Jieli Geng ${ }^{3}$, Meijie Qu' ${ }^{1}$ Fang Yuan², Yuyang Wang ${ }^{1}$, Jiaji Pan², Yongfang Li ${ }^{1}$, Yuanyuan Ma', Panting Zhou', Zhijun Zhang ${ }^{2}$ and Guo-Yuan Yang ${ }^{1,2}$

\begin{abstract}
Blood-brain barrier damage is a critical pathological feature of ischemic stroke. Oligodendrocyte precursor cells are involved in maintaining blood-brain barrier integrity during the development. However, whether oligodendrocyte precursor cell could sustain blood-brain barrier permeability during ischemic brain injury is unknown. Here, we investigate whether oligodendrocyte precursor cell transplantation protects blood-brain barrier integrity and promotes ischemic stroke recovery. Adult male ICR mice $(n=68)$ underwent 90 min transient middle cerebral artery occlusion. After ischemic assault, these mice received stereotactic injection of oligodendrocyte precursor cells $\left(6 \times 10^{5}\right)$. Oligodendrocyte precursor cells transplantation alleviated edema and infarct volume, and promoted neurological recovery after ischemic stroke. Oligodendrocyte precursor cells reduced blood-brain barrier leakage via increasing claudin-5, occludin and $\beta$-catenin expression. Administration of $\beta$-catenin inhibitor blocked the beneficial effects of oligodendrocyte precursor cells. Wnt7a protein treatment increased $\beta$-catenin and claudin- 5 expression in endothelial cells after oxygen-glucose deprivation, which was similar to the results of the conditioned medium treatment of oligodendrocyte precursor cells on endothelial cells. We demonstrated that oligodendrocyte precursor cells transplantation protected blood-brain barrier in the acute phase of ischemic stroke via activating Wnt/ $\beta$-catenin pathway. Our results indicated that oligodendrocyte precursor cells transplantation was a novel approach to the ischemic stroke therapy.
\end{abstract}

\section{Introduction}

Blood-brain barrier (BBB) disruption is a critical pathological feature in the acute phase of cerebral disorder $^{1,2}$. BBB consists of brain endothelial cells with their tight junctions, the basement membrane, pericytes, and astrocyte end-feet, which shields the brain against toxins and pathogens, and allows delivery of nutrients to the brain $^{3,4}$. Brain endothelial cells are the core element of

\footnotetext{
Correspondence: Jieli Geng (gengjieli@renji.com) or

Zhijun Zhang (zhangzj@sjtu.edu.cn) or Guo-Yuan Yang (gyyang@sjtu.edu.cn) ${ }^{1}$ Department of Neurology, Ruijin Hospital, School of Medicine, Shanghai Jiao Tong University, Shanghai 200025, China

${ }^{2}$ Med-X Research Institute and School of Biomedical Engineering, Shanghai

Jiao Tong University, Shanghai 200030, China

Full list of author information is available at the end of the article.

Edited by A. Verkhratsky
}

$\mathrm{BBB}^{5}$. Tight junction proteins of brain endothelial cells determine the BBB permeability ${ }^{2}$. Brain endothelial cells express high level of tight junction proteins including claudins, occludin, and zonula occludens family ${ }^{6,7}$. Claudin-5, the most abundant claudin of BBB, disappears in endothelial cells from 1 day to 7 days after ischemic stroke and reappears in newly repaired brain endothelial cells, which makes itself as a promising target to protect $\mathrm{BBB}$ injury $^{2}$. Studies reported $\mathrm{Wnt} / \beta$-catenin pathway played a central role in the tight junction protein formation ${ }^{1,3,6}$. Activation of $\mathrm{Wnt} / \beta$-catenin pathway could upregulate tight junction proteins expression especially claudin-5.

Stem cell transplantation showed a promising potential for ischemic stroke therapy ${ }^{8-11}$. Oligodendrocyte precursor cells (OPCs) derived from the ventricular zone in 
the embryo and migrated widely through the central nervous system, comprising about $5 \%$ of cells in the adult brain $^{12,13}$. OPCs transplantation could enhance spatial learning and memory after hypoxic ischemic injury in premature rat brain ${ }^{14}$. Researches implied OPCs could maintain the $\mathrm{BBB}$ integrity and had close interaction with brain endothelial cells during development ${ }^{15}$. OPCs migrated along the vasculature via CXCl12-Wnt-CXCR4 signaling $^{13}$. OPC HIF $1 / 2 \alpha$ activity could induce paracrine Wnt7 to promote Wnt-dependent angiogenesis ${ }^{16}$. Meanwhile OPCs were a source of Wnt7, which served as the dominant ligands acting on frizzled receptors (Fzd) on brain endothelial cells and then activated $\beta$-catenin ${ }^{17}$. This evidence intrigued us to hypothesize that OPCs transplantation could attenuated tight junction disruption in brain endothelial cells after cerebral ischemia.

In this study, we aim to explore: (1) whether OPCs transplantation promotes the brain function recovery in ischemic mice; (2) whether OPCs transplantation attenuates $\mathrm{BBB}$ breakdown and reduces brain edema at the acute phase of cerebral ischemia; and (3) whether $\mathrm{Wnt} / \beta$-catenin pathway is involved in the effect of OPCs on endothelial cells.

\section{Results}

OPCs identification, transplantation, and differentiation

Cultured OPCs showed a bipolar or multipolar morphology under phase-contrast microscope (Fig. 1a).
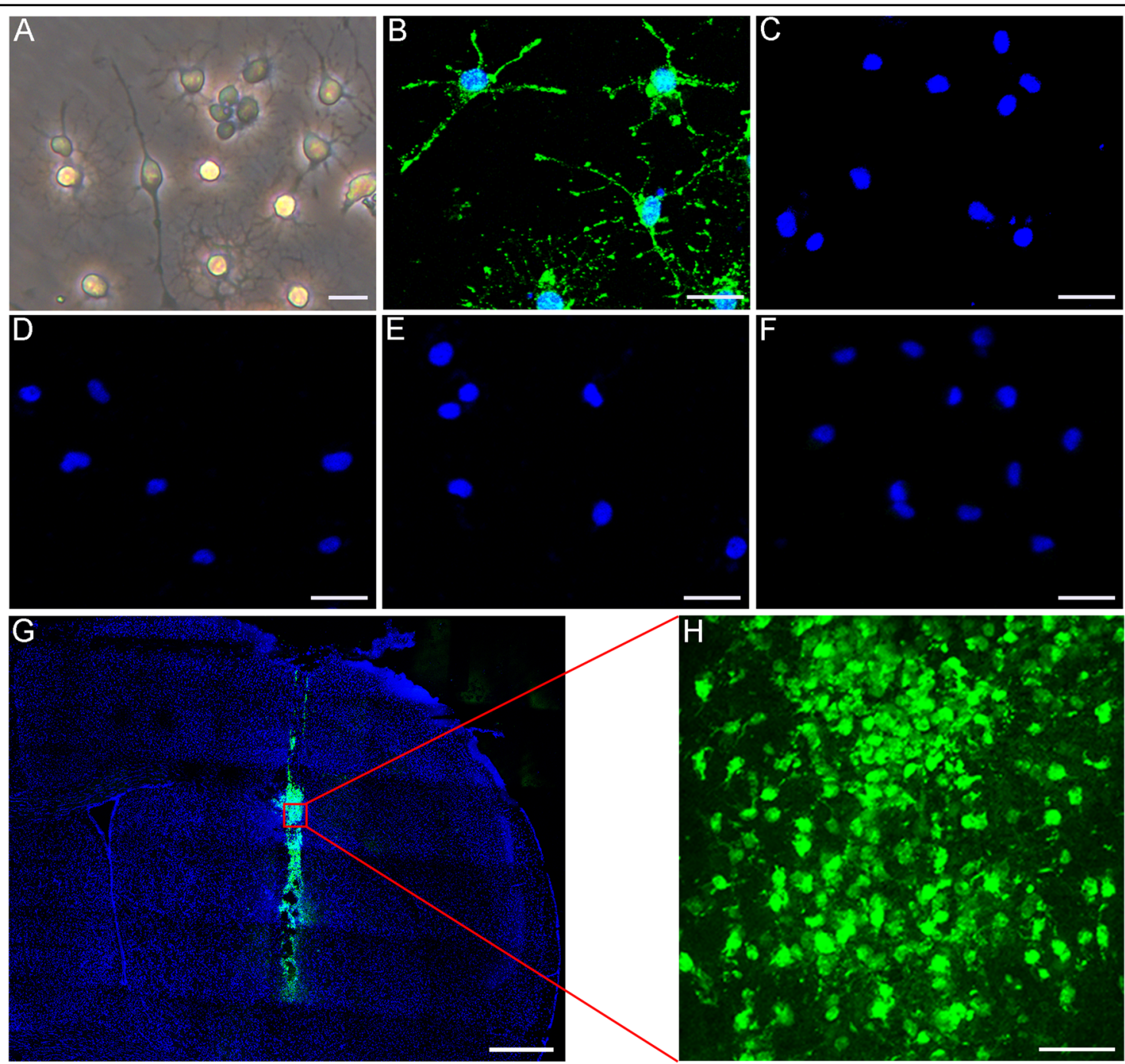

Fig. 1 OPC identification and transplantation. a Cultured cells under phase-contrast microscopy. Immunofluorescence staining depicted that cultured cells were positive for NG2 (b), and negative for MBP (c), GFAP (d), NeuN (e), and Iba-1 (f). g Green fluorescent OPCs (CFDA-SE stained) were located in the ischemic hemisphere after 3 days of injection. $\mathbf{h}$ Survival of OPCs after injection. Scale bar $=10 \mu \mathrm{m}(\mathbf{a}), 25 \mu \mathrm{m}(\mathbf{b}-\mathbf{f}), 500 \mu \mathrm{m}(\mathbf{g})$, and $50 \mu \mathrm{m}(\mathbf{h})$ 
Immunofluorescent staining showed that the percentage of $\mathrm{NG}^{+}$cells was $93.82 \%$ (Fig. $1 \mathrm{~b}$ and Supplementary Fig. $1 E)$. Very few cells expressed GFAP, Iba-1, MBP or NeuN (Fig. 1c-f, the positive controls in Supplementary Fig. $1 \mathrm{~A}-\mathrm{D})$. For in vivo cell tracking, we labeled OPCs with CFDA-SE. The results demonstrated that a considerable number of transplanted OPCs could survive after 3 days following middle cerebral artery occlusion (MCAO) (Fig. 1g, h).

OPCs transplantation attenuated infarct volume and brain edema after MCAO

The brain edema and infarct volume were evaluated using cresyl violet staining. Results showed that the edema formation was significantly less in the OPC-treated mice compared with the phosphate buffered saline (PBS)treated mice at 3 days after MCAO (Fig. 2a, $n=8-10$ per group, $p<0.05)$. Infarct volume was also significantly decreased in the Stroke + OPC group compared with the Stroke + PBS group at 3 days after MCAO (Fig. 2a, $p<0.05$ ).

\section{OPCs transplantation improved neurobehavioral recovery after MCAO}

We used modified neurological severity score (mNSS) and rotarod test to evaluate the neurological function. OPCs transplantation significantly decreased neurological scores at 3, 7, and 14 days after MCAO (Fig. 2b, $n=8-10$ per group, $p<0.05)$. Rotarod tests showed that the time staying on the rotarod was prolonged in the Stroke + OPC group compared with the Stroke + PBS group at 7 and 14 days after MCAO (Fig. 2c, $n=8-10$ per group, $p<$ $0.05)$. Results demonstrated that neurobehavioral deficiency was attenuated in the Stroke + OPC group compared with the Stroke + PBS group. Cognitive defect was detected by step through test at 12 days after MCAO. Step through test showed that transplanted OPCs ameliorated the cognitive damage caused by ischemic stroke (Fig. $2 \mathrm{~d}$, $n=8-10$ per group, $p<0.001$ ).

OPCs transplantation sustained BBB integrity after MCAO

To evaluate $\mathrm{BBB}$ permeability after ischemic brain injury, IgG protein extravasation were measured. IgG staining showed that IgG protein leaked into brain tissue after ischemia. The quantification result showed that leaked IgG protein decreased in the Stroke + OPC group compared with the Stroke + PBS group at 3 days after MCAO (Fig. 3a, $p<0.05$ ), which indicated that BBB integrity was protected through the OPC transplantation.

To investigate the mechanism of $\mathrm{BBB}$ disruption, we analyzed the expression of claudin- 5 and occludin in cerebral vascular structures using claudin-5 and occludin/ CD31 double staining. Results showed that OPCs alleviated the disruption of claudin-5 (Fig. 3b, $p<0.01$ ).
Occludin expression presented gap on the endothelial cell margin of cerebral microvessel after ischemic injury. Our results suggested that OPCs greatly reduced gap formation after MCAO (Fig. 3c, $p<0.001$ ).

\section{OPCs transplantation enhanced the $\beta$-catenin expression in endothelial cells}

To examine whether OPCs had beneficial effect on endothelial cells after MCAO, we performed $\beta$-catenin/ CD31 double staining. $\beta$-catenin was mainly expressed on the cerebral vasculature (Fig. 4a). The quantification result indicated OPCs transplantation upregulated the expression of $\beta$-catenin (Fig. 4b, $p<0.001$ ).

\section{Inhibition of $\beta$-catenin aggravated claudin-5 disruption, BBB leakage, and neurological outcomes}

To further examine whether $\beta$-catenin was involved in the beneficial role of OPCs after ischemic stroke, $\beta$-catenin inhibitor XAV-939 was injected in OPC-treated ischemic mice for consecutive 3 days. Axin 2 can degrade $\beta$-catenin and XAV-939 can stabilize Axin2 to promote $\beta$-catenin degradation ${ }^{16}$. We found that XAV-939 could block the beneficial effect of OPCs on neurological deficiency, infarct volume, and edema at 3 days after MCAO (Fig. 5a-c, $p<0.05$ ). XAV-939 treatment reversed the protective role of OPCs on the integrity of BBB (Fig. $5 \mathrm{~d}$, $p<0.05)$. IgG leakage was increased after XAV-939 administration. Immunostaining images showed $\beta$-catenin level decreased in the endothelial cells of OPCtreated with XAV-939 mice (Fig. 5e, $p<0.001$ ). The expression of claudin-5 was also decreased after XAV-939 administration (Fig. 5f, $p<0.05$ ).

\section{Wnt7a secreted by OPCs activated $\beta$-catenin and upregulated claudin- 5 in bEnd. 3 cells after oxygen-glucose deprivation (OGD)}

Western blot revealed Wnt7a level was increased in the Stroke + OPC group compared with the Stroke + PBS group (Fig. 6a, b, $p<0.05$ ). Images analysis indicated both the conditioned medium (CM) of OPC and Wnt7a could increase $\beta$-catenin expression in bEnd.3 cells compared with the control (Fig. 6c, $p<0.01$ ). Claudin-5 expression increased in CM of OPC $(p<0.01)$ and Wnt7a $(p<0.05)$ groups compared with the control group in bEnd. 3 cells at $24 \mathrm{~h}$ after OGD (Fig. $6 \mathrm{~d}$ ). While knocking down the Wnt7a of OPC could block the effect of CM of OPC.

\section{CM of OPC and Wnt7a supported the endothelial barrier after OGD}

To confirm whether CM of OPC and Wnt7a could support the endothelial barrier, in vitro endothelial permeability assay was performed (Fig. 6e). The leaked amount of Evans blue was detected for the evaluation of 

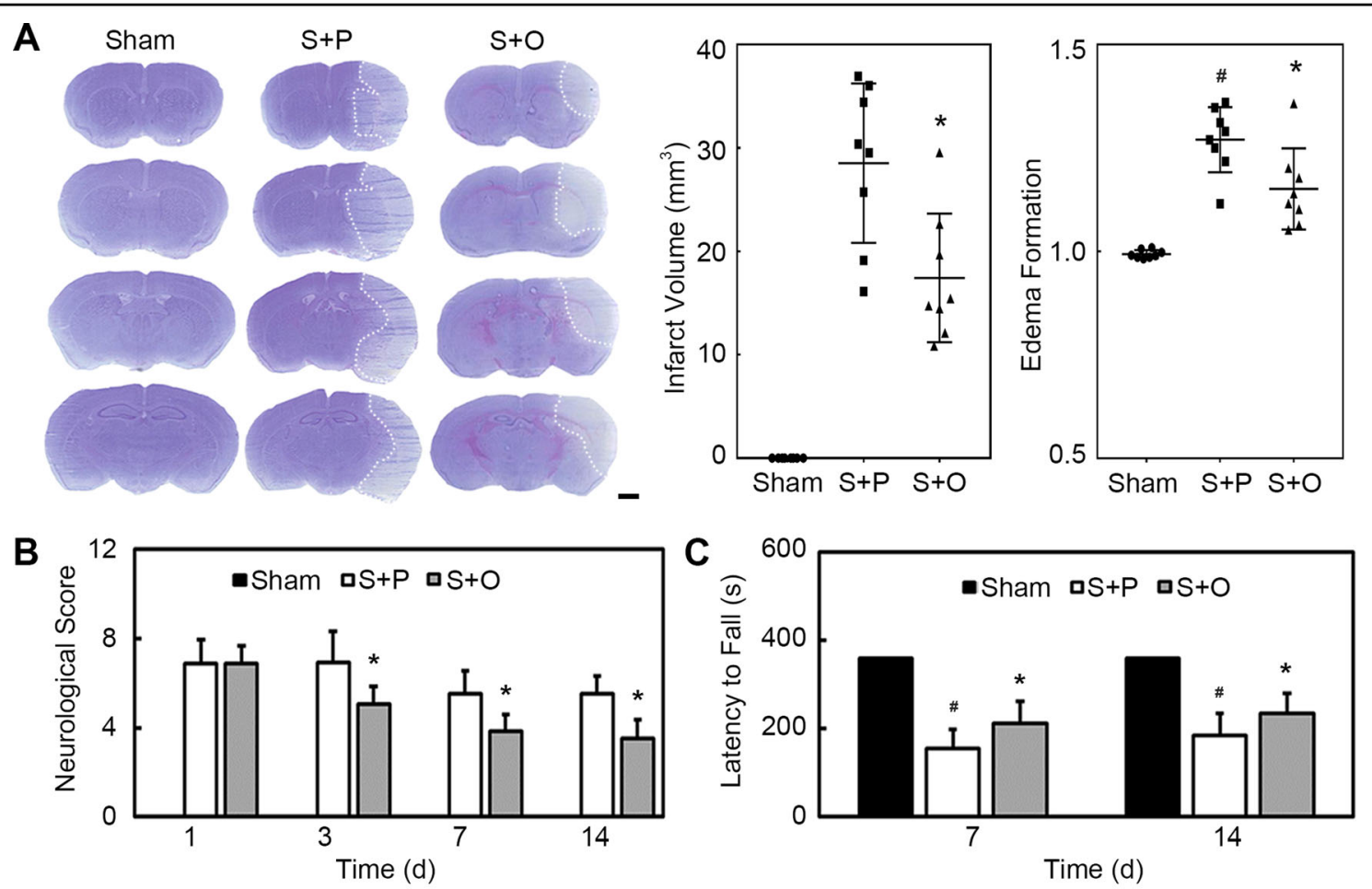

D
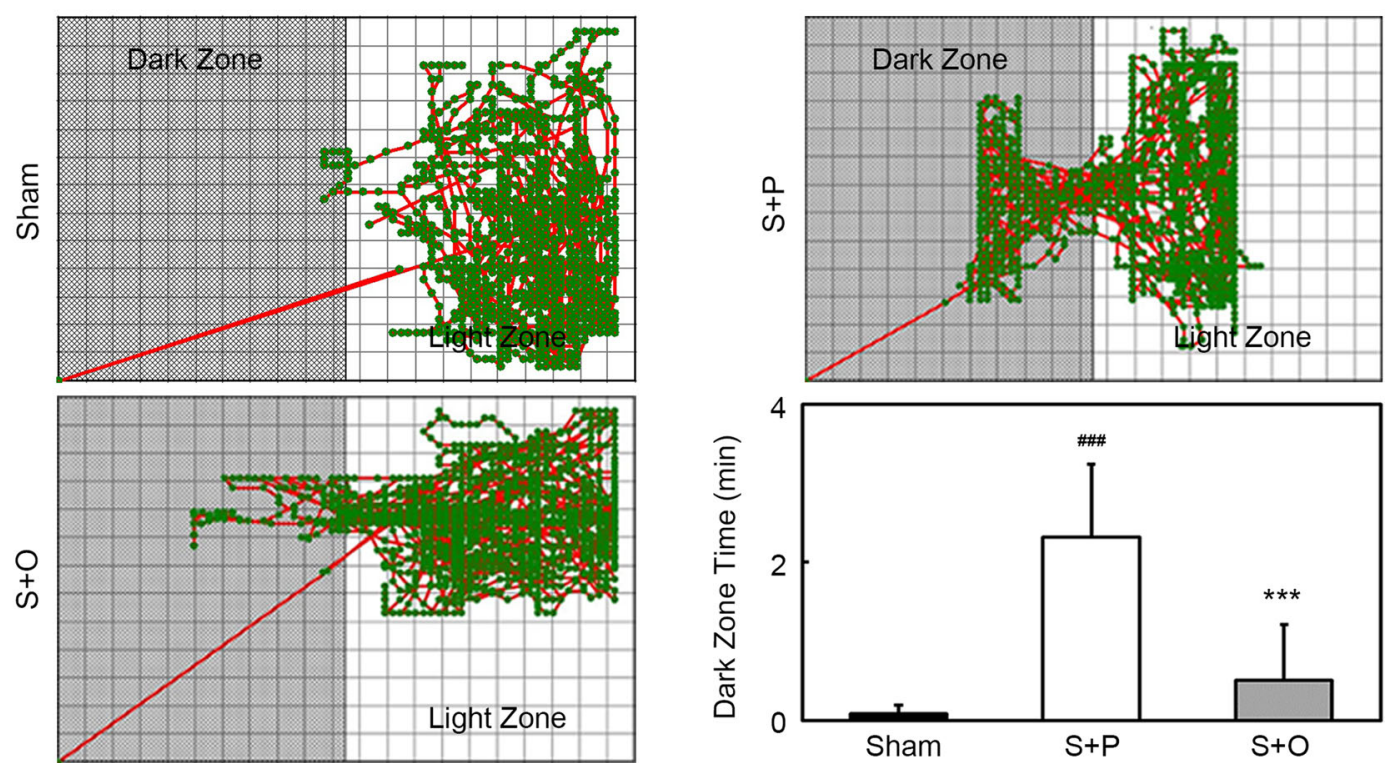

Fig. 2 OPCs transplantation attenuated infarct volume and brain edema and improved neurological outcomes after MCAO. a Cresyl violet staining showed the infarction after MCAO in Sham, Stroke + PBS and Stroke + OPC groups. Bar graphs indicated that OPCs transplantation reduced infarct volume and edema formation. Scale bar $=1 \mathrm{~mm}$. b Bar graph of neurological scores. $\mathbf{c}$ Bar graph of latency to fall. $\mathbf{d}$ Trace pictures and the bar graph of step through test showed that OPCs ameliorated the cognitive damage caused by ischemic stroke. Data are mean $\pm S D, n=8-10$ per group. ${ }^{*} p<0.05,{ }^{* * *} p<0.001$, as compared with Stroke + PBS group. ${ }^{\#} p<0.05,{ }^{\# \# \#} p<0.001$, as compared with Sham group. S + P: Stroke + PBS; $\mathrm{S}+\mathrm{O}:$ Stroke + OPC.

permeability. The Evans blue result showed that both CM of OPC and Wnt7a decreased the endothelial permeability compared with the control and CM of OPC with knocking down Wnt7a (Fig. 6f, $p<0.001$ ).

\section{Discussion}

Our study demonstrated that OPCs transplantation effectively protected BBB integrity and promoted functional recovery in ischemic mice. Wnt/ $\beta$-catenin pathway 


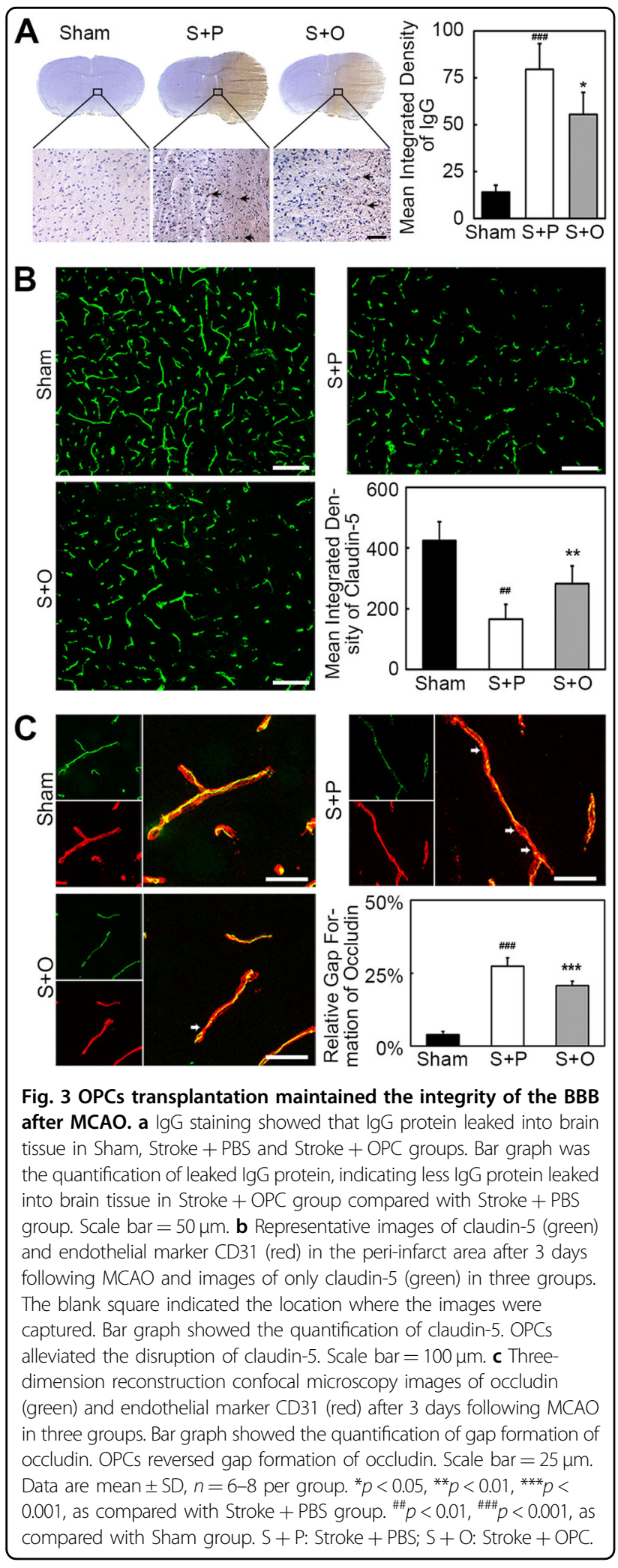

activated by OPC treatment participated in the upregulation of tight junction proteins after ischemic stroke.
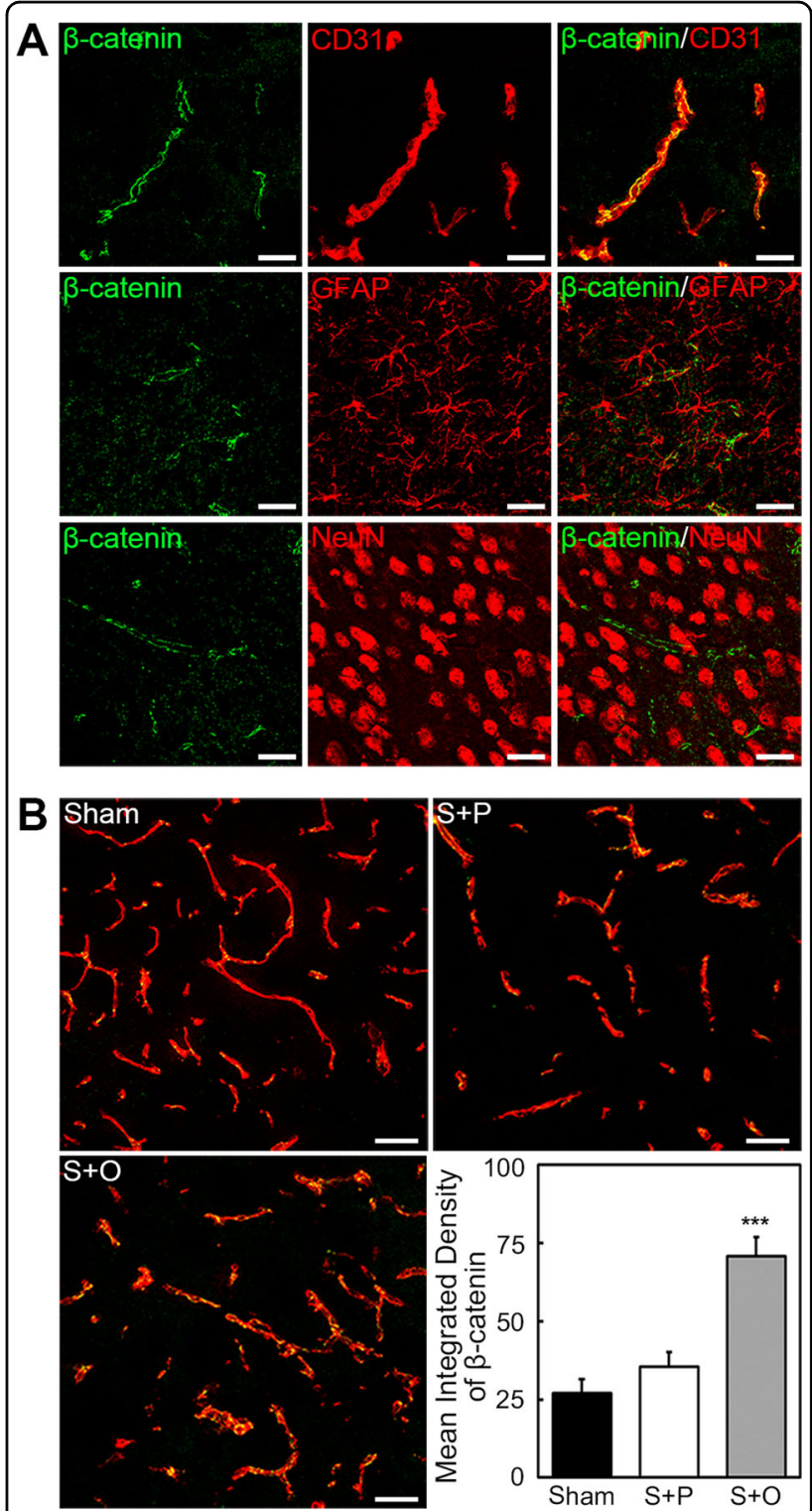

Fig. 4 OPCs transplantation enhanced the expression of $\beta$-catenin in endothelial cells. a $\beta$-catenin (green) was mainly expressed on the cerebrovasculature (red). Astrocytes $\left(\mathrm{GFAP}^{+}\right)$and neuron $\left(\mathrm{NeuN}^{+}\right)$expressed little $\beta$-catenin. Scale bar $=25 \mu \mathrm{m}$.

b Images of $\beta$-catenin (green) and endothelial marker CD31 (red) in the peri-infarct area after 3 days following MCAO in Sham, Stroke + PBS, and Stroke + OPC groups. Bar graph showed OPCs upregulated the expression of $\beta$-catenin. Data are mean $\pm S D, n=6-8$ per group, ${ }^{* * *} p<0.001$, as compared with Stroke + PBS group. Scale bar $=$ $50 \mu \mathrm{m} . \mathrm{S}+\mathrm{P}$ : Stroke + PBS; S + O: Stroke + OPC.

BBB disruption is a canonical feature of ischemic stroke $^{18}$. Oxidation and inflammation exacerbate the loss of tight junction proteins such as claudin- 5 and occludin in the microvasculature, and further increase BBB permeability ${ }^{19,20}$. Our data showed that OPCs transplantation significantly attenuated infarct volume and brain 

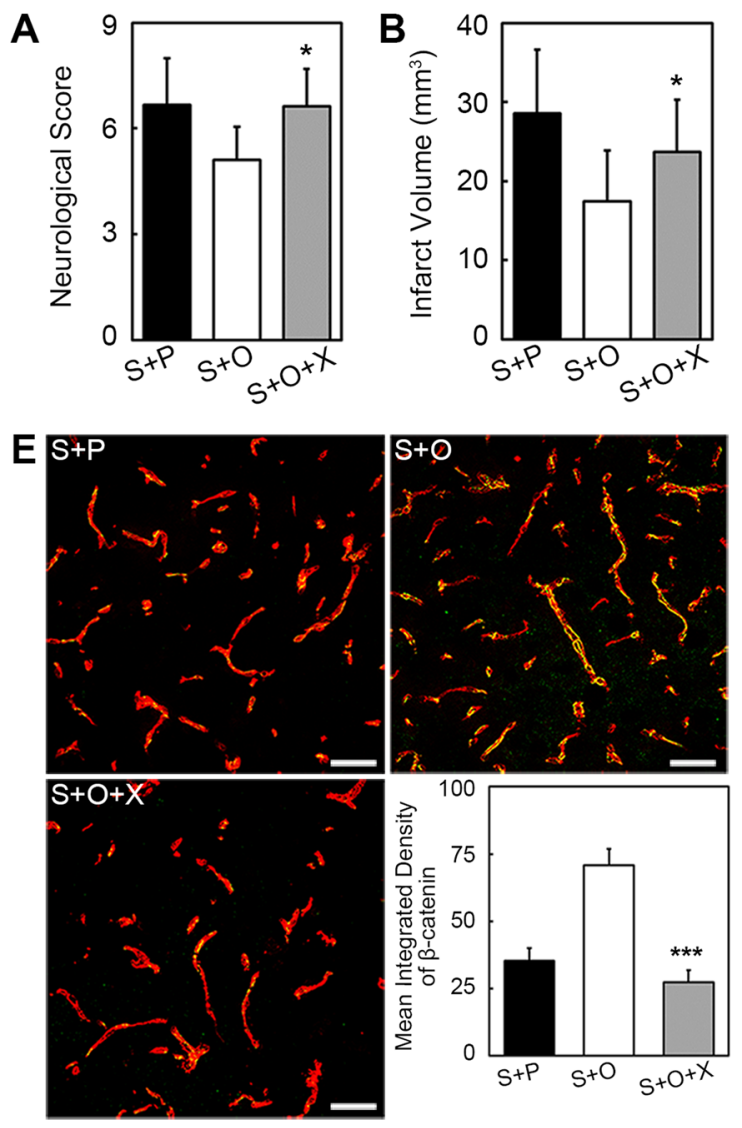
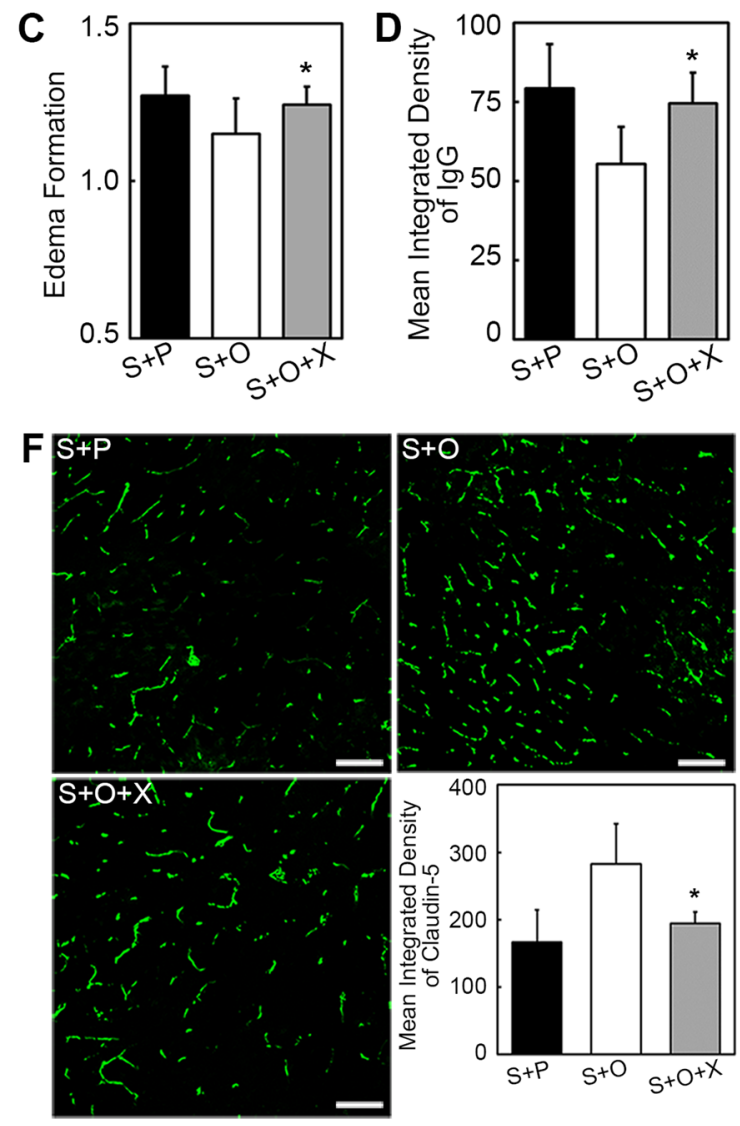

Fig. 5 Inhibition of $\beta$-catenin aggravated the neurological outcomes, infarct volume, edema formation, and BBB disruption. Bar graphs indicated the inhibition of $\beta$-catenin hindered the beneficial effects of OPCs in neurological scores (a), infarct volume (b), and edema formation (c), respectively. $\mathbf{d}$ IgG staining showed the IgG leakage in Stroke + PBS, Stroke + OPC, and Stroke + OPC + XAV-939 groups after 3 days following MCAO. e Images showed the $\beta$-catenin (green)/CD31(red) immunofluorescence staining in above three groups. XAV-939 decreased the expression of $\beta$-catenin in endothelial cells. $\mathbf{f}$ Immunofluorescent images of claudin-5 (green) and bar graph displayed XAV-939 reversed the claudin-5 upregulation caused by OPCs. Data are mean \pm SD, $n=6-8$ per group, ${ }^{*} p<0.05,{ }^{* *} p<0.001$, as compared with Stroke + OPC group. Scale bar $=50 \mu m(\mathbf{e})$ and $100 \mu \mathrm{m}(\mathbf{f}) . \mathrm{S}+\mathrm{P}$ : Stroke + PBS; S + O: Stroke + OPC; S + O + X: Stroke + OPC + XAV-939.

edema after ischemia. OPCs transplantation reduced IgG leakage, rescued claudin-5 disruption in ischemic stroke, which suggested that OPCs transplantation protected $\mathrm{BBB}$ integrity through reducing tight junction protein degradation. Meanwhile, OPCs transplantation ameliorated neurobehavioral deficiency and cognitive defects, which may be due to the alleviating of BBB damage.

OPCs migrated along the vasculature and Wnt signaling regulated OPC-endothelium interaction during CNS development ${ }^{13}$. Our results supported that transplanted OPCs upregulated the $\beta$-catenin expression in endothelial cells. The inhibition of $\beta$-catenin by XAV-939 blocked the beneficial effects of OPCs on neurological outcomes, infarct volume, and edema formation. Furthermore, the inhibition of $\beta$-catenin reversed the protective role of OPCs on the integrity of BBB and aggravated claudin- 5 disruption. The level of claudin- 5 had positive correlation with that of $\beta$-catenin. The result supported that OPCs transplantation increased the expression of claudin-5 via upregulating $\beta$-catenin in endothelial cells.

Previous study reported that OPCs could secret large amounts of Wnt7a, which served as the dominant ligands in Wnt signaling and acted on its receptor Fzd on brain endothelial cells $s^{16,17,21}$. Wnt ligands produced by immature neuroectodermal cells could bind to Fzd on brain endothelial cells and contribute to the induction of claudin $-5^{22,23}$. We found that both $\mathrm{CM}$ of OPC and Wnt7a could upregulated $\beta$-catenin expression in bEnd.3 cells. Meanwhile, claudin-5 expression in bEnd. 3 cells also increased in CM of OPC and Wnt7a groups. This indicated CM of OPC and Wnt7a upregulated claudin-5 via Wnt/ $\beta$-catenin signaling. The beneficial effect of $\mathrm{CM}$ of OPC might be largely due to Wnt7a.

Besides Wnt7a, OPCs can secret trophic factors, such as IGF1, GDNF, and TGF- $\beta$, providing trophic signals to neighboring cells ${ }^{15,24}$. These trophic factors may also be 

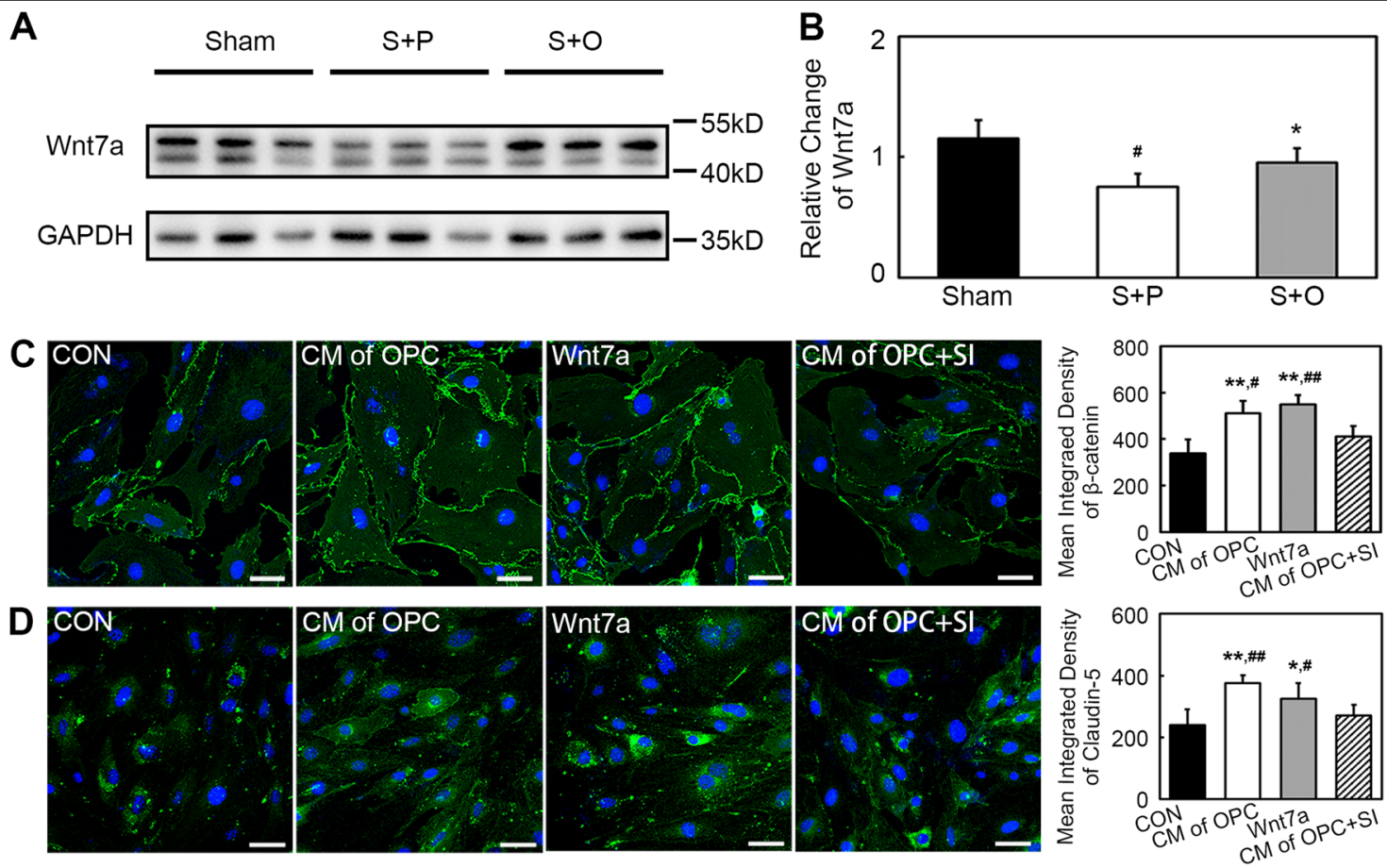

E
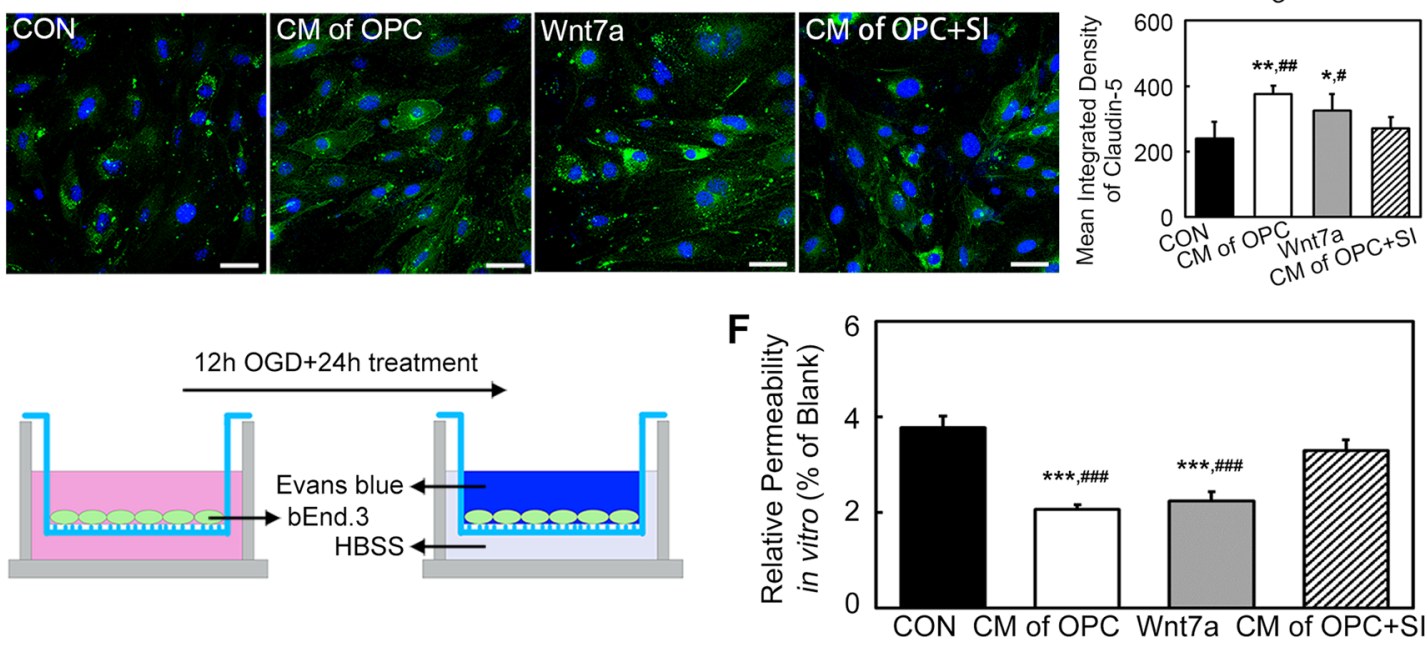

Fig. 6 Wnt7a secreted by OPCs activated $\beta$-catenin and upregulated claudin- 5 in bEnd. 3 cells after OGD. a Western blot of Wnt7a in Sham, Stroke + PBS, and Stroke + OPC groups. b Western blot revealed Wnt7a increased in Stroke + OPC group after ischemia compared with Stroke + PBS group. Data are mean $\pm S D, n=3$ per group, ${ }^{*} p<0.05$, as compared with Stroke + PBS group, ${ }^{*} p<0.05$, as compared with Sham group. $S+P:$ Stroke + PBS; $S+O$ : Stroke + OPC. $\mathbf{c}$ Images showed the $\beta$-catenin (green) expression of bEnd.3 cells in control (CON), CM of OPC, Wnt7a, and CM of OPC + SI groups at $24 \mathrm{~h}$ after OGD. Statistical analysis indicated CM of OPC and Wnt7a could promote $\beta$-catenin expression in bEnd. 3 cells compared with control. Data are mean $\pm S D, n=4$ per group, ${ }^{* *} p<0.01$, as compared with CON group, ${ }^{\#} p<0.05,{ }^{\# \#} p<0.01$, as compared with CM of OPC + SI group. $\mathbf{d}$ Images showed the claudin-5 (green) expression of bEnd. 3 cells in CON, CM of OPC, Wnt7a, and CM of OPC + SI groups at $24 \mathrm{~h}$ after OGD. Data are mean \pm SD, $n=4$ per group, ${ }^{*} p<0.05,{ }^{* *} p<0.01$, as compared with CON group, ${ }^{\#} p<0.05,{ }^{\# \#} p<0.01$, as compared with CM of OPC + SI group. e Experimental setup for Evans blue determination in vitro. $\mathbf{f}$ Bar graph indicated the relative permeability of endothelial barrier in vitro. Data are mean $\pm S D, n=4$ per group, ${ }^{* * *} p<0.001$, as compared with CON group, ${ }^{\# \# \#} p<0.001$, as compared with CM of OPC + SI group. Scale bar $=50 \mu \mathrm{m}$.

possible to regulate tight junction in brain endothelial cells. The cross talk between OPCs and brain endothelial cells regulate the $\mathrm{BBB}^{15,24}$. Present study supported the following possible mechanism between OPCs and brain endothelial cells: in the normal condition, endothelial cells expressed tight junction proteins such as claudin-5 and occludin, which played an important role in the integrity of BBB. In ischemic stroke, the tight junction proteins could be degraded. Transplanted OPCs released Wnt7a, which acting on the corresponding receptor Fzd in the membrane of endothelial cells, then $\mathrm{Wnt} / \beta$-catenin pathway was activated and upregulated claudin-5 (Fig. 7).
In conclusion, we demonstrated that OPCs transplantation promoted stroke recovery in ischemic mice. OPCs protected BBB in the acute phase of cerebral ischemia via activating $\mathrm{Wnt} / \beta$-catenin pathway of endothelial cells. OPCs transplantation is a novel approach to the ischemic stroke therapy.

\section{Materials and methods \\ Experimental protocol}

Animal procedures and protocols were approved by the Institutional Animal Care and Use Committee of Shanghai Jiao Tong University, Shanghai, China. All studies 


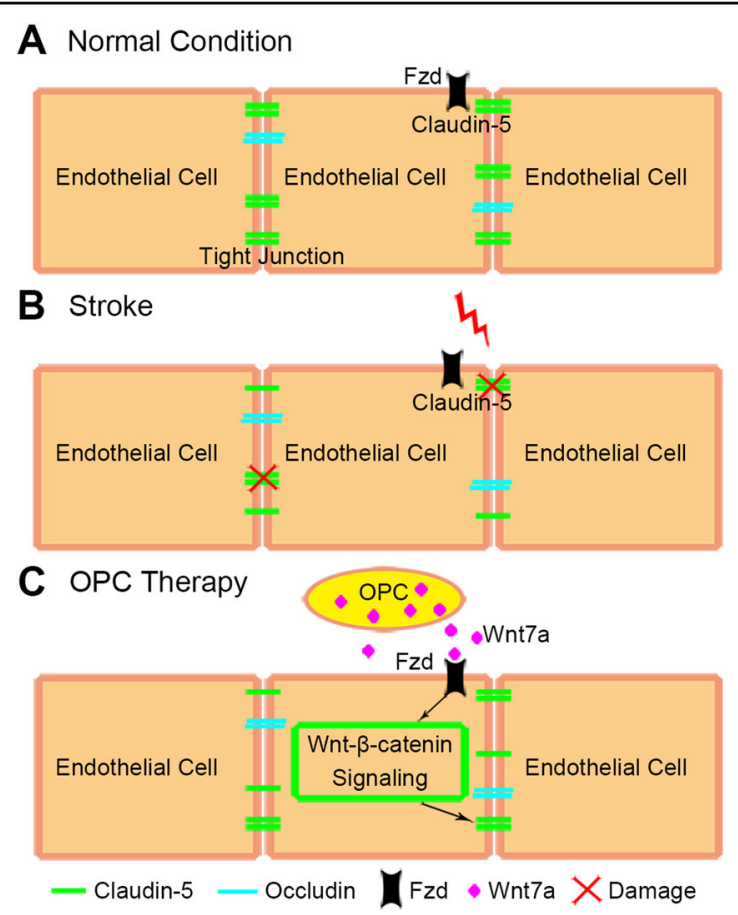

Fig. 7 The schematic diagram showed the possible mechanism of OPC therapy for stroke. a In the normal condition, endothelial cells expressed tight junction proteins such as claudin-5 and occludin to keep the integrity of BBB. $\mathbf{b}$ The tight junction proteins were disrupted by oxidative stress and inflammation after stroke. $\mathbf{c}$ In the OPC therapy, transplanted OPCs released Wnt7a which acting on the corresponding receptor Fzd in the membrane of endothelial cells. Then Wnt/ $\beta$-catenin pathway was activated and upregulated claudin-5.

were conducted in accordance with the US National Research Council's Guide for the Care and Use of Laboratory Animals, the US Public Health Service's Policy on Humane Care and Use of Laboratory Animals and Guide for the Care and Use of Laboratory Animals. Animal studies were reported according to ARRIVE guidelines. Sixty-eight adult male ICR mice weighing 28-30 g (JSJ, Shanghai, China) were used in the study. Animals were fed normally in the SPF proved animal facility in Shanghai Jiao Tong University, China. Mice were randomly divided into four groups: stroke + OPC group, stroke + OPC + XAV-939 group, stroke + PBS group, and sham group, $n=8-16$ per group.

\section{OPCs isolation and identification}

The brain cortex was dissected from P1 Sprague-Dawley rat pups as described ${ }^{25}$. Brain tissue was dissociated into a single-cell suspension by trypsin at $37^{\circ} \mathrm{C}$ for $10 \mathrm{~min}$. After the suspension was filtered with a $70-\mu \mathrm{m}$ filter, cells were seeded on poly-d-lysine (PDL, Sigma, St. Louis, MO) coated culture flasks in DMEM (Gibco, Carlsbad, CA) with $10 \%$ fatal calf serum (Gibco). Eight to ten days later, the microglia were separated from glia cell mixtures after $30 \mathrm{~min}$ of culture by a $220 \mathrm{rpm}$ shake and then OPCs were collected by $20 \mathrm{~h}$ of culture by a $200 \mathrm{rpm}$ shake. Collected cells were injected into the mouse striatum or seeded on a PDL-coated culture dish in Neurobasal-A (Gibco) containing 2\% B27 (Gibco), $10 \mathrm{ng} / \mathrm{ml}$ PDGF-AA (Gibco), $10 \mathrm{ng} / \mathrm{ml}$ bFGF (Peprotech, Rocky Hill, NJ), and $2 \mathrm{mmol} / \mathrm{l}$ glutamine (Gibco).

For identification, cells were fixed with $4 \%$ paraformaldehyde and blocked by $10 \%$ bovine serum albumin. Then OPCs were incubated with primary antibodies against PDGFR- $\alpha$ (1:100, Santa Cruz Biotechnology, Santa Cruz, CA), NG2 (1:200, Millipore, Bedford, MA), GAFP (1:200, Millipore), MBP (1:200, Abcam, Cambridge, UK), NeuN (1:200, Millipore), and Iba-1 (1:200, WAKO, Osaka, Japan) at $4{ }^{\circ} \mathrm{C}$ overnight. Cells were incubated with the fluorescence conjugated second antibodies $37^{\circ} \mathrm{C}$ for $1 \mathrm{~h}$.

\section{Mouse model of transient MCAO}

Transient MCAO was performed as described previously $^{26,27}$. Mice were anesthetized by ketamine/xylazine (100 mg/kg, Fujian Gutian Pharmaceutical Co., Ltd, Gutian, China)/xylazine (10 mg/kg, Sigma) and placed in the supine position. After the isolation of the left common carotid artery, external carotid artery, and internal carotid artery, the origin of middle cerebral artery was occluded by a silicone-coated 6-0 suture (Covidien, Mansfield, MA). Reperfusion was performed after $90 \mathrm{~min}$ of MCAO by withdrawing the suture. The success of occlusion was assessed by a laser Doppler flowmetry (Moor Instruments, Devon, UK) with a decrease of cerebral blood flow at least $80 \%$ of the baseline. Sham mice were conducted the same procedure except the insertion of suture.

\section{OPCs transplantation and inhibition of $\beta$-catenin}

Animals were randomly divided into following groups as Stroke + PBS, Stroke + OPC, and Stroke + OPC + XAV-939. OPCs or PBS (as a control) were injected at $24 \mathrm{~h}$ after MCAO. Before transplantation, OPCs were labeled with carboxyfluorescein diacetate-succinimidyl ester (CFDA-SE, Beyotime, Shanghai, China) for the tracking. Mice were anesthetized and received stereotaxic transplantation. A microsurgical drill made a small skull hole $2 \mathrm{~mm}$ lateral to the bregma. An amount of $6 \times 10^{5}$ OPCs was suspected in $5 \mu \mathrm{l}$ PBS and slowly injected into the left striatum at $2 \mathrm{~mm}$ lateral to the bregma and $3 \mathrm{~mm}$ under the dura $(\mathrm{AP}=0 \mathrm{~mm}, \mathrm{ML}=2 \mathrm{~mm}, \mathrm{DV}=3 \mathrm{~mm})$ at a rate of $1 \mu \mathrm{l} / \mathrm{min}$ by the $10 \mu \mathrm{l}$ Hamilton syringe (Hamilton, Bonaduz, Switzerland). The same amount of PBS was injected as control. For the OPC plus XAV-939 group, XAV-939 (40 mg/kg, MCE, Monmouth Junction, NJ) was injected intraperitoneally once a day ${ }^{16,28}$. The mice were injected i.p. daily with cyclosporine A ( $5 \mathrm{mg} / \mathrm{kg}$, Sigma) for immunosuppression after cell transplantation. 


\section{Neurobehavioral function examination}

An investigator who was blinded to the experimental treatment performed mNSS to evaluate the neurological function at 1 day, 3 days, 7 days, and 14 days after $\mathrm{MCAO}^{29}$. The mNSS ranged from 0 to 14 and included motor, sensory, balance, and reflex tests.

Rotarod test was performed at 7 and 14 days after MCAO. Mice were trained on a rotating rod at $20 \mathrm{rpm}$ for 3 consecutive days before MCAO. Mice were placed on the rod for adaption, after which the rod was continuously accelerated to $40 \mathrm{rpm}$. The time mice stayed on the rod (latency to fall) was recorded.

Step through test was performed at 12 and 13 days after MCAO. At the first day of the test, mice were put into the light space of smart cage (AfaSci Research Laboratories, Redwood City, CA) with an electric stimulator at the base. The mouse received an electric shock once it stepped into the dark box. After $24 \mathrm{~h}$, mice were put into the smart cage again without electric stimulation and monitored for $10 \mathrm{~min}$. The trace was recorded by the infrared detector and analyzed by the smart cage software.

\section{Measurement of brain infarct volume and edema formation}

Mice were sacrificed at 3 days after MCAO and brains were cut into a series of $20 \mu \mathrm{m}$ thick coronal sections. We performed cresyl violet staining (Sigma) to measure the brain infarct volume and edema formation. Infarct volume was calculated by ImageJ software (National Institutes of Health, Bethesda, MD) as described previously ${ }^{18,26}$. Edema formation was calculated by the ipsilateral volume divided by contralateral volume.

\section{BBB permeability assay}

The leakage of IgG was detected to assess BBB permeability $^{18,27}$. Briefly, brain slices were fixed with $4 \%$ paraformaldehyde and incubated with Universal $A B C$ Kit (Vector Labs, Burlingame, CA). After that, the immunoreactivity was visualized by DAB reagents (Vector Labs) and slices were counterstained with hematoxylin. We selected four slices from anterior to posterior coronal coordinates in each mouse for the statistical analysis. Three fields along the ischemic penumbra area for each slice were randomly collected and analyzed with ImageJ software (National Institutes of Health) for mean integrated density analysis.

\section{Immunohistochemistry determination}

Brain slices were fixed with methanol at $4{ }^{\circ} \mathrm{C}$ for $10 \mathrm{~min}$ and blocked with diluted donkey serum (Jackson ImmunoResearch, West Grove, PA) for $60 \mathrm{~min}$ at room temperature. Slides were incubated with primary antibodies of CD31 (1:200, R\&D system, Minneapolis, MN), occludin
(1:200, Life Technologies, Carlsbad, CA), claudin-5 (1:200, Life Technologies), $\beta$-catenin (1:100, Abcam), GFAP (1:500, Millipore), NeuN (1:50, Millipore), and Wnt7a (1:100, Abcam) overnight at $4{ }^{\circ} \mathrm{C}$. After rinsing three times with PBS, brain slices were incubated with the fluorescence conjugated second antibodies for $1 \mathrm{~h}$ at room temperature. Immunofluorescence photos were collected by a confocal microscope (Leica, Solms, Germany). For claudin- 5 and $\beta$-catenin quantification, mean integrated density was measured by ImageJ software (National Institutes of Health). For occludin staining, the gap length was presented as percentage (\%) of whole tight junction staining ${ }^{26,27}$. We randomly chose at least four vessels in the peri-focal region per brain section, and total eight sections per animal. Then we measured the length of vessel and gap by ImageJ software (National Institutes of Health). Gap length was presented as percentage (\%) of gap length in whole vessel.

\section{Real-time PCR and western blot analysis}

The real-time PCR assay and western blot were performed as described previously ${ }^{26}$. The two stage RT-PCR amplification parameters were $95^{\circ} \mathrm{C}$ for $30 \mathrm{~s}$ followed by 40 cycles of $95^{\circ} \mathrm{C}$ for $5 \mathrm{~s}$ and $60^{\circ} \mathrm{C}$ for $30 \mathrm{~s}$. Wnt7a mRNA expression level was normalized to reference gene GAPDH and displayed as relative expression of mRNA by $2^{-\Delta c t}$ method. Wnt7a forward primer was CTC GCC ATT AGA AAA GTT CC, reverse primer was AAG CCT CTT ATC CAG TGG TT. GAPDH forward primer was GAT GGT GAA GGT CGG TGT GA, the reverse primer was TGA ACT TGC CGT GGG TAG AG.

For western blot analysis, the membranes were blocked with $5 \%$ skim milk and incubated with primary antibodies against Wnt7a (1:500, Abcam) and GAPDH (1:1000, Abcam) overnight at $4{ }^{\circ} \mathrm{C}$. After washing with TBST buffer, the membranes were incubated with HRPconjugated secondary antibody.

\section{Wnt7a siRNA interference in OPCs}

Sequences of interfering siRNA segments were as follows: 5-GCCUUCACCUAUGCAAUUATT-3. Nontargeting siRNA with scramble served as a negative control (NC).

OPCs were seeded at a density of $5 \times 10^{5}$ cells per well in six-well plates. After $24 \mathrm{~h}$, the $\mathrm{CM}$ was collected. Twenty micromolars FAM-siRNA $(8 \mu \mathrm{l}$, Genepharma, Shanghai, China) and $3.75 \mu$ l Lipofectamine ${ }^{\circledR} 3000$ (Invitrogen) were each diluted by $125 \mu$ l Opti-MEM (Gibco). Diluted siRNA and Lipofectamine ${ }^{\circledR} 3000$ were mixed and added into the $1750 \mu \mathrm{l}$ culture medium of OPCs. After $24 \mathrm{~h}$ of interference, we changed new culture medium and after $48 \mathrm{~h}$ of interference, the $\mathrm{CM}$ and cell proteins of three groups (Blank, NC and siRNA) were collected. 


\section{bEnd.3 cell culture and OGD experiment}

bEnd. 3 cells were purchased from the China Center for Type Culture Collection (Wuhan, China) and were grown in DMEM (Gibco) with 10\% fetal bovine serum (FBS, Gibco). In OGD, bEnd.3 cells were seeded in six-well plates at a density of $2 \times 10^{5}$ cells per well and incubated with FBS and glucose free DMEM at $5 \% \mathrm{CO}_{2}$ and $95 \% \mathrm{~N}_{2}$ atmosphere using an airtight chamber for $12 \mathrm{~h}$. Then the medium was changed with 70\% normal DMEM with 30\% Neurobasal-A (control), CM of OPC, CM of OPC with Wnt7a siRNA (CM of OPC + SI), or Neurobasal-A with Wnt7a protein $(50 \mathrm{ng} / \mathrm{mL}, \mathrm{R} \& D \text { system })^{30}$. After $24 \mathrm{~h}$, bEnd.3 cells were performed immunostaining to test claudin- 5 and $\beta$-catenin expression.

\section{In vitro BBB permeability assay}

bEnd. 3 cells were plated on PDL-coated 3- $\mu$ m pore size transwell chambers of 12-well plates (Corning, New York, $\mathrm{NY}$ ) at a density of $2 \times 10^{5}$ cells per well. When cells reached confluency, they were treated with OGD for $12 \mathrm{~h}$. Then bEnd. 3 cells were treated with 70\% normal DMEM with $30 \%$ Neurobasal-A (control), CM of OPC, CM of $\mathrm{OPC}+\mathrm{SI}$ or Neurobasal-A with Wnt7a protein $(50 \mathrm{ng} /$ $\mathrm{mL}, \mathrm{R} \& \mathrm{D}$ system) for $24 \mathrm{~h}$.

In Evans blue determination, the upper and bottom chambers were washed with Hank's balanced salt solution (HBSS). Evans blue staining solution was 0.25\% Evans blue (Sigma) and $4 \%$ bovine serum albumin in HBSS. HBSS $(1.5 \mathrm{ml})$ was added to the bottom chamber, and Evans blue staining solution $(0.5 \mathrm{ml})$ was added to the upper chamber. Then the plate was incubated at $37^{\circ} \mathrm{C}$ for $5 \mathrm{~min}$. The solution in the bottom chamber was collected, and the amount of Evans blue was quantified at $610 \mathrm{~nm}$ by a spectrometer (Biotek, Winooski, VT) ${ }^{19,31}$. The chamber without bEnd. 3 cells was as the blank group. The permeability was presented as the percentage compared with the blank group.

\section{Statistical analysis}

Sample size was determined according to our previous publications for similar outcomes ${ }^{18,26,27}$. Samples size was estimated using a type I error rate of 0.05 and a power of 0.8 on a two-sided test by power analysis. Analysis were performed by SPSS. Parametric data were analyzed using one-way ANOVA followed by Tukey's post hoc test. Data were expressed as mean $\pm \mathrm{SD}$. A probability value less than 0.05 was considered significant.

\footnotetext{
Acknowledgements

This study was supported by grants from the Scientific Research and Innovation Program of Shanghai Education Commission (2019-01-07-00-02E00064, G.Y.Y.), the National Natural Science Foundation of China (81771251, G. Y.Y.; 81771244, Z.J.Z.; 81471178 , G.Y.Y.), the National Key Research and Development Program of China (2016YFC1300600), K. C. Wong Education Foundation (G.Y.Y.), and the Science and Technology Commission of Shanghai Municipality (17ZR1413600, Z.J.Z.).
}

\begin{abstract}
Author details
${ }^{1}$ Department of Neurology, Ruijin Hospital, School of Medicine, Shanghai Jiao Tong University, Shanghai 200025, China. ${ }^{2}$ Med-X Research Institute and School of Biomedical Engineering, Shanghai Jiao Tong University, Shanghai 200030, China. ${ }^{3}$ Department of Neurology, Renji Hospital, School of Medicine, Shanghai Jiao Tong University, Shanghai 200025, China
\end{abstract}

\section{Conflict of interest}

The authors declare that they have no conflict of interest.

\section{Publisher's note}

Springer Nature remains neutral with regard to jurisdictional claims in published maps and institutional affiliations.

Supplementary Information accompanies this paper at (https://doi.org/ 10.1038/s41419-019-2206-9).

Received: 26 August 2019 Revised: 6 December 2019 Accepted: 9 December 2019

Published online: 06 January 2020

\section{References}

1. Chang, J. et al. Gpr124 is essential for blood-brain barrier integrity in central nervous system disease. Nat. Med. 23, 450-460 (2017).

2. $L V$, J. et al. Focusing on claudin-5: a promising candidate in the regulation of BBB to treat ischemic stroke. Prog. Neurobiol. 161, 79-96 (2018).

3. Obermeier, B., Daneman, R. \& Ransohoff, R. M. Development, maintenance and disruption of the blood-brain barrier. Nat. Med. 19, 1584-1596 (2013).

4. Engelhardt, B. \& Liebner, S. Novel insights into the development and maintenance of the blood-brain barrier. Cell Tissue Res. 355, 687-699 (2014).

5. Lim, R. G. et al. Huntington's disease iPSC-derived brain microvascular endothelial cells reveal WNT-mediated angiogenic and blood-brain barrier deficits. Cell Rep. 19, 1365-1377 (2017).

6. Tietz, S. \& Engelhardt, B. Brain barriers: Crosstalk between complex tight junctions and adherens junctions. J. Cell Biol. 209, 493-506 (2015).

7. Ren, $C$. et al. Limb ischemic perconditioning attenuates blood-brain barrier disruption by inhibiting activity of MMP-9 and occludin degradation after focal cerebral ischemia. Aging Dis. 6, 406-417 (2015).

8. Liu, X. et al. Cell based therapies for ischemic stroke: from basic science to bedside. Prog. Neurobiol. 115, 92-115 (2014).

9. Tang, Y. H., Ma, Y. Y., Zhang, Z. J., Wang, Y. T. \& Yang, G. Y. Opportunities and challenges: stem cell-based therapy for the treatment of ischemic stroke. CNS Neurosci. Ther. 21, 337-347 (2015).

10. Zhang, $\mathrm{H}$. et al. Combining injectable plasma scaffold with mesenchymal stem/stromal cells for repairing infarct cavity after ischemic stroke. Aging Dis. 8, 203-214 (2017).

11. Dongsheng, $\mathrm{H}$. et al. Proteomic analysis of the peri-infarct area after human umbilical cord mesenchymal stem cell transplantation in experimental stroke. Aging Dis. 7, 623-634 (2016).

12. Levine, J. M., Reynolds, R. \& Fawcett, J. W. The oligodendrocyte precursor cell in health and disease. Trends Neurosci. 24, 39-47 (2001).

13. Tsai, H. H. et al. Oligodendrocyte precursors migrate along vasculature in the developing nervous system. Science 351, 379-384 (2016).

14. Chen, L. X. et al. Neuroprotective effects of oligodendrocyte progenitor cell transplantation in premature rat brain following hypoxic-ischemic injury. PLOS ONE 10, e0115997 (2015).

15. Seo, J. H. et al. Oligodendrocyte precursor cells support blood-brain barrier integrity via TGF-beta signaling. PLOS ONE 9, e103174 (2014).

16. Yuen, T. J. et al. Oligodendrocyte-encoded HIF function couples postnatal myelination and white matter angiogenesis. Cell 158, 383-396 (2014).

17. Cho, C., Smallwood, P. M. \& Nathans, J. Reck and Gpr124 are essential receptor cofactors for Wnt7a/Wnt7b-specific signaling in mammalian CNS angiogenesis and blood-brain barrier regulation. Neuron 95, 1221-1225 (2017).

18. Huang, J. et al. CXCR4 antagonist AMD3100 protects blood-brain barrier integrity and reduces inflammatory response after focal ischemia in mice. Stroke 44, 190-197 (2013). 
19. Podjaski, $C$. et al. Netrin 1 regulates blood-brain barrier function and neuroinflammation. Brain 138, 1598-1612 (2015).

20. Al Ahmad, A., Gassmann, M. \& Ogunshola, O. O. Involvement of oxidative stress in hypoxia-induced blood-brain barrier breakdown. Microvasc. Res. 84 222-225 (2012).

21. Liebner, S. \& Plate, K. H. Differentiation of the brain vasculature: the answer came blowing by the Wnt. J. Angiogenes Res. 2, 1 (2010).

22. Zhou, Y. et al. Canonical WNT signaling components in vascular development and barrier formation. J. Clin. Investig. 124, 3825-3846 (2014).

23. Zhou, Y. \& Nathans, J. Gpr124 controls CNS angiogenesis and blood-brain barrier integrity by promoting ligand-specific canonical wnt signaling. Dev. Cell 31, 248-256 (2014)

24. Miyamoto, N. et al. Crosstalk between cerebral endothelium and oligodendrocyte. Cell Mol. Life Sci. 71, 1055-1066 (2014).

25. Chen, Y. et al. Isolation and culture of rat and mouse oligodendrocyte precursor cells. Nat. Protoc. 2, 1044-1051 (2007)
26. Geng, J. et al. Endothelial progenitor cells transplantation attenuated bloodbrain barrier damage after ischemia in diabetic mice via HIF-1alpha. Stem Cell Res. Ther. 8, 163 (2017).

27. Tang, G. et al. Mesenchymal stem cells maintain blood-brain barrier integrity by inhibiting aquaporin-4 upregulation after cerebral ischemia. Stem Cells $\mathbf{3 2}$, 3150-3162 (2014)

28. Bai, J. et al. Epigenetic downregulation of SFRP4 contributes to epidermal hyperplasia in psoriasis. J. Immunol. 194, 4185-4198 (2015).

29. Li, Y. et al. Postacute stromal cell-derived factor-1alpha expression promotes neurovascular recovery in ischemic mice. Stroke 45, 1822-1829 (2014).

30. von Maltzahn, J., Renaud, J. M., Parise, G. \& Rudnicki, M. A. Wnt7a treatment ameliorates muscular dystrophy. Proc. Natl Acad. Sci. USA 109, 20614-20619 (2012).

31. Lin, H. C. et al. From the cover: comparative proteomics reveals silver nanoparticles alter fatty acid metabolism and amyloid beta clearance for neuronal apoptosis in a triple cell coculture model of the blood-brain barrier. Toxicol. Sci. 158, 151-163 (2017). 